\title{
Coloring the edges of a random graph without a monochromatic giant component*
}

\author{
Reto Spöhel ${ }^{\dagger}$ \\ Institute of Theoretical Computer Science \\ ETH Zürich, Switzerland \\ rspoehel@inf.ethz.ch
}

\author{
Angelika Steger \\ Institute of Theoretical Computer Science \\ ETH Zürich, Switzerland \\ asteger@inf .ethz.ch
}

\author{
Henning Thomas ${ }^{\ddagger}$ \\ Institute of Theoretical Computer Science \\ ETH Zürich, Switzerland \\ hthomas@inf .ethz.ch
}

Submitted: Mar 25, 2010; Accepted: Sep 19, 2010; Published: Oct 5, 2010

Mathematics Subject Classification: 05C80, 05C15

\begin{abstract}
We study the following two problems: i) Given a random graph $G_{n, m}$ (a graph drawn uniformly at random from all graphs on $n$ vertices with exactly $m$ edges), can we color its edges with $r$ colors such that no color class contains a component of size $\Theta(n)$ ? ii) Given a random graph $G_{n, m}$ with a random partition of its edge set into sets of size $r$, can we color its edges with $r$ colors subject to the restriction that every color is used for exactly one edge in every set of the partition such that no color class contains a component of size $\Theta(n)$ ?

We prove that for any fixed $r \geqslant 2$, in both settings the (sharp) threshold for the existence of such a coloring coincides with the known threshold for $r$-orientability of $G_{n, m}$, which is at $m=r c_{r}^{*} n$ for some analytically computable constant $c_{r}^{*}$. The fact that the two problems have the same threshold is in contrast with known results for the two corresponding Achlioptas-type problems.
\end{abstract}

\section{Introduction}

One of the most prominent areas of research in random graph theory is the component structure of the random graph $G_{n, m}$ (a graph drawn uniformly at random from all graphs

${ }^{*}$ A preliminary version of this paper appeared in the proceedings of the European Conference on Combinatorics, Graph Theory and Applications (EuroComb) 2009 [17].

${ }^{\dagger}$ The author was supported by the Swiss National Science Foundation, grant 200020-119918. He is now at MPI Saarbrücken.

${ }^{\ddagger}$ The author was supported by the Swiss National Science Foundation, grant 200021-120284. 
on $n$ vertices with exactly $m$ edges). In their seminal 1960 paper, Erdös and Rnyi [10] discovered that an abrupt 'phase transition' occurs at $m=n / 2$. We say that an event occurs asymptotically almost surely (a.a.s.) if it occurs with probability tending to 1 as $n$ tends to infinity. For the sake of readability, we omit ceiling and floor signs whenever they are not essential. In particular, we write $G_{n, m}$ where technically we should write $G_{n,\lfloor m\rfloor}$ (or $G_{n,\lceil m\rceil}$ ) since $m(n)$ is not necessarily an integer.

Theorem 1 ([10]) For any constant $c>0$ the following holds.

- If $c<0.5$, then a.a.s. all components of $G_{n, c n}$ have $\mathcal{O}(\log n)$ vertices.

- If $c>0.5$, then a.a.s. $G_{n, c n}$ contains one component with $\Theta(n)$ vertices, and all other components have $\mathcal{O}(\log n)$ vertices.

We can also think of this result in the setting of a random graph process where random edges are inserted one by one into an initially empty graph. Then the theorem states that a.a.s. a linear-sized 'giant component' emerges quite precisely at the point where the average degree in the graph is 1 . In the past decades, much research has been devoted to more detailed studies of the behaviour of the random graph (process) around this critical point; see $[7,14]$ for an overview of results.

A direction of research that has appeared more recently in the literature concerns the question whether the emergence of a giant component can be postponed or accelerated if some freedom of choice is introduced in the setup. The most prominent model of this type is the so-called Achlioptas process, in which two random edges are sampled at each step, and one of them has to be selected to be included in the graph. Note that this is an online model in which we are required to select edges before we have seen the entire random graph. As it turns out, the emergence of a giant component can be both accelerated or slowed down by a constant factor if appropriate edge selection strategies are used $[2,4,6,12,16]$. However, no threshold result similar to Theorem 1 is known for this or other online scenarios.

Motivated by this line of research, Bohman and Kim [5] studied a similar question in an offline setting. Given $c n$ pairs of random edges, can we select one edge from each pair to obtain a graph with $c n$ edges that does not contain a giant component? If we assume that all edges are sampled without replacement, then drawing $c n$ random edge pairs is equivalent to drawing $2 \mathrm{cn}$ random edges and partitioning these into pairs uniformly at random. More generally, we denote for any fixed integer $r \geqslant 2$ by $G_{n, m}^{r}$ a random $r$ matched graph obtained by generating a random graph $G_{n, m}$ and partitioning its edge set into sets of size $r$ uniformly at random (where we assume w.l.o.g. that $m$ is divisible by $r$ ). Throughout, we call these $r$-sets and denote them by $E_{j}, 1 \leqslant j \leqslant m / r$. We say that a subgraph is an Achlioptas subgraph of $G_{n, m}^{r}$ if it contains exactly one edge from each $r$-set.

In this terminology, the result of Bohman and Kim reads as follows.

Theorem $2([5])$ There exists an analytically computable constant $c_{1} \approx 0.9768$ such that for any constant $c>0$ the following holds. 
- If $c<c_{1}$, then a.a.s. $G_{n, 2 c n}^{2}$ has an Achlioptas subgraph (with cn edges) all components of which have $\mathcal{O}\left(n^{1-\varepsilon}\right)$ vertices, where $\varepsilon>0$ is a constant depending on $c$.

- If $c>c_{1}$, then a.a.s. every Achlioptas subgraph of $G_{n, 2 c n}^{2}$ contains a component with $\Theta(n)$ vertices.

A similar but less restrictive scenario was investigated earlier by Bohman, Frieze, and Wormald [4]. Given an ordinary random graph $G_{n, 2 c n}$, can we select $c n$ edges such that the resulting graph does not contain a giant component? As it turns out, the threshold for this property is slightly higher than the one stated in Theorem 2.

Theorem $3([4])$ There exists an analytically computable constant $c_{2} \approx 0.9793$ such that for any constant $c>0$ the following holds.

- If $c<c_{2}$, then a.a.s. $G_{n, 2 c n}$ has a subgraph with cn edges all components of which have $\mathcal{O}(1)$ vertices.

- If $c>c_{2}$, then a.a.s. every subgraph of $G_{n, 2 c n}$ with cn edges contains a component with $\Theta(n)$ vertices.

In this paper we consider analogous questions for two Ramsey-type settings. In the first we are given a random graph $G_{n, m}$ and are required to color its edges with a fixed number $r$ of available colors. Our goal is to avoid a monochromatic giant component in each of the $r$ color classes. Note that, by the pigeon-hole principle, Theorem 3 yields an upper bound for the case $r=2$.

The second setting we investigate is more restrictive and motivated by Theorem 2 . We call an $r$-edge-coloring of the random $r$-matched graph $G_{n, m}^{r}$ valid if every color is used for exactly one edge from every $r$-set. Note that that this implies in particular that every color class has the same size. Our goal now is to find a valid $r$-edge-coloring of $G_{n, m}^{r}$ such that no color class contains a giant component. Note that, again by the pigeon-hole principle, Theorem 2 yields an upper bound for the case $r=2$ in this more restricted setting that is slightly better than the bound given by Theorem 3 for the first setting.

As it turns out (see Theorem 4 below), the thresholds of these two Ramsey settings coincide, in contrast to what happens for the edge-selection scenarios studied in Theorem 2 and Theorem 3. In order to state our main result, we need to introduce one more notion. For any integer $r \geqslant 2$, a graph $G$ is called $r$-orientable if there exists an orientation of its edges such that the in-degree of every vertex is at most $r$. Cain, Sanders, and Wormald [8] and independently Fernholz and Ramachandran [11] showed that the property that $G_{n, m}$ is $r$-orientable has a sharp threshold at $m=r c_{r}^{*} n$ for some analytically computable constant $c_{r}^{*}$. In [8] the following numerical values are stated: $c_{2}^{*} \approx 0.897, c_{3}^{*} \approx 0.959$, $c_{4}^{*} \approx 0.980$, and $c_{5}^{*} \approx 0.989$.

Our result is the following.

Theorem 4 For every integer $r \geqslant 2$, let $c_{r}^{*}$ denote the constant which determines the threshold for $r$-orientability of the random graph $G_{n, r c n}$. Then for any constant $c>0$ the following holds. 
- If $c<c_{r}^{*}$, then a.a.s. there exists a valid $r$-edge-coloring of $G_{n, r c n}^{r}$ in which all monochromatic components have $\mathcal{O}\left(n^{1-\varepsilon}\right)$ vertices, where $\varepsilon>0$ is a constant depending on $c$ and $r$.

- If $c>c_{r}^{*}$, then a.a.s. every $r$-edge-coloring of $G_{n, r c n}$ contains a monochromatic component with $\Theta(n)$ vertices.

Note that the first statement of the theorem is about the restricted setting and the second statement is about the unrestricted setting. Together they imply that the thresholds of both scenarios coincide.

The threshold for the unrestricted setting was also derived independently from us by Bohman, Frieze, Krivelevich, Loh, and Sudakov [3]. They also investigated the corresponding online setting, where random edges appear one by one and have to be colored immediately with one of $r$ available colors, and the version of the problem where the goal is to create monochromatic giants instead of avoiding them.

Let us conclude this introduction by stating an open question we would like to see answered: What is the threshold for the property that the vertices of a random graph can be colored in such a way that no color class induces a linear-sized component? It is not hard to see that for any $c>1.5$, a random 3-vertex-coloring of $G_{n, c n}$ yields a giant component a.a.s. On the other hand, Achlioptas and Molloy [1] showed that a.a.s. $G_{n, c n}$ can be properly 3 -colored as long as $c<1.923$, thus providing a significantly better lower bound than a random vertex-coloring.

\section{Proof of Theorem 4}

\subsection{Preliminaries}

We state two results which we will need in our proofs. The following density lemma is a straightforward generalisation of Lemma 2 in [4].

Lemma 5 ([4]) Let $c>0$. For every $\varepsilon>0$ there exists $\delta=\delta(c, \varepsilon)>0$ such that a.a.s. the random graph $G:=G_{n, c n}$ has the property that for every $S \subseteq V(G)$ for which $G[S]$ contains more than $(1+\varepsilon)|S|$ edges we have $|S| \geqslant \delta n$.

The following lemma conveniently sums up the arguments given in Section 4 of [5].

Lemma 6 ([5]) Let $c>0$. For every $\delta>0$ there exists $\varepsilon=\varepsilon(c, \delta)>0$ such that a.a.s. the random graph $G:=G_{n, c n}$ has the property that for every subgraph $H \subseteq G$ in which all components are trees or unicyclic, a.a.s. the graph obtained by removing $\min \{\delta n, e(H)\}$ edges uniformly at random from $H$ has only components with $\mathcal{O}\left(n^{1-\varepsilon}\right)$ vertices. 


\subsection{Upper bound proof for unrestricted setting}

Let $c>c_{r}^{*}$. We need to show that a.a.s. every $r$-edge-coloring of $G_{n, r c n}$ contains a monochromatic component of linear size.

In $[8,11]$ it was shown that $m=r c_{r}^{*} n$ - the threshold for $r$-orientability of $G_{n, m}-$ coincides with the threshold for the property that the $(r+1)$-core of $G_{n, m}$ has average degree at most $2 r$ (the $k$-core of a graph $G$ is the maximal subgraph of $G$ with minimum degree at least $k$ ). For some details, see the introduction of [11]. It is known [15] that a.a.s. the $(r+1)$-core of $G_{n, r c_{r}^{*} n}$ has linear size, which implies by a standard two-round exposure argument that for $c>c_{r}^{*}$, there exists $\varepsilon>0$ such that a.a.s. the $(r+1)$-core $C_{r+1}$ of $G_{n, r c n}$ has average degree at least $(1+\varepsilon) 2 r$, i.e., satisfies

$$
\left|E\left(C_{r+1}\right)\right| \geqslant(1+\varepsilon) r\left|V\left(C_{r+1}\right)\right| .
$$

(For explicit statements about the number of vertices and edges in cores of random graphs see $[9,13])$. By averaging, a.a.s. in every $r$-edge-coloring of $G_{n, r c n}$ there exists a monochromatic connected subgraph $H \subseteq C_{r+1}$ with at least $(1+\varepsilon)|V(H)|$ edges. By Lemma 5 such a subgraph is a.a.s. of linear size.

\subsection{Lower bound proof for restricted setting}

Let $c<c_{r}^{*}$. We need to show that a.a.s. we can find a valid $r$-edge-coloring of $G_{n, r c n}^{r}$ in which every monochromatic component is of size $\mathcal{O}\left(n^{1-\varepsilon}\right)$ for some constant $\varepsilon$ which only depends on $c$ and $r$.

Similarly to the proof of Theorem 2 in [5], we generate $G_{n, r c n}^{r}$ by first generating a slightly denser random $r$-matched graph $G^{+}$and then removing a few $r$-sets uniformly at random. Let $\delta=\delta(c, r)>0$ such that $c+\delta<c_{r}^{*}$, and let $G^{+}:=G_{n, r(c+\delta) n}^{r}$ be a random $r$ matched graph with $r$-sets $E_{1}, \ldots, E_{(c+\delta) n}$. We let $G$ denote the $r$-matched graph obtained by removing $\delta n r$-sets chosen uniformly at random from $G^{+}$. Note that, by symmetry, $G$ is distributed exactly as $G_{n, r c n}^{r}$. Thus if we can show that a.a.s. there is a valid $r$-edgecoloring of $\mathrm{G}^{+}$in which all monochromatic components are trees or unicyclic, the lower bound part of Theorem 4 immediately follows by applying Lemma 6 (with $c \leftarrow r(c+\delta$ ) and $\delta \leftarrow \delta$ ) in each color class and taking a union bound over all $r$ color classes.

Recall that $m=r c_{r}^{*} n$ is the threshold for $r$-orientability of the random graph $G_{n, m}$. Hence, by our choice of $c$, a.a.s. there exists an orientation of the underlying unmatched graph of $G^{+}$in which every vertex has in-degree at most $r$. Conditioning on this and considering a fixed such orientation $d$, we now look for a partition of $G^{+}$into $r$ edgedisjoint Achlioptas subgraphs such that in every color the in-degree of every vertex is at most 1 . Note that every edge $e \in E\left(G^{+}\right)$is oriented towards exactly one vertex and is contained in exactly one of the $r$-sets $E_{1}, \ldots, E_{(c+\delta) n}$. This naturally defines a bipartite multigraph $B$ with parts $V\left(G^{+}\right)$and $\left\{E_{1}, \ldots, E_{(c+\delta) n}\right\}$ in which every edge of $G^{+}$induces an edge connecting the vertex it is oriented towards with the $r$-set it is contained in (see Figure 1 for an example). Clearly, every $r$-set $E_{j}$ has degree exactly $r$ in $B$, and, since $d$ is an $r$-orientation of $G^{+}$, every vertex $v \in V\left(G^{+}\right)$has degree at most $r$ in $B$. 

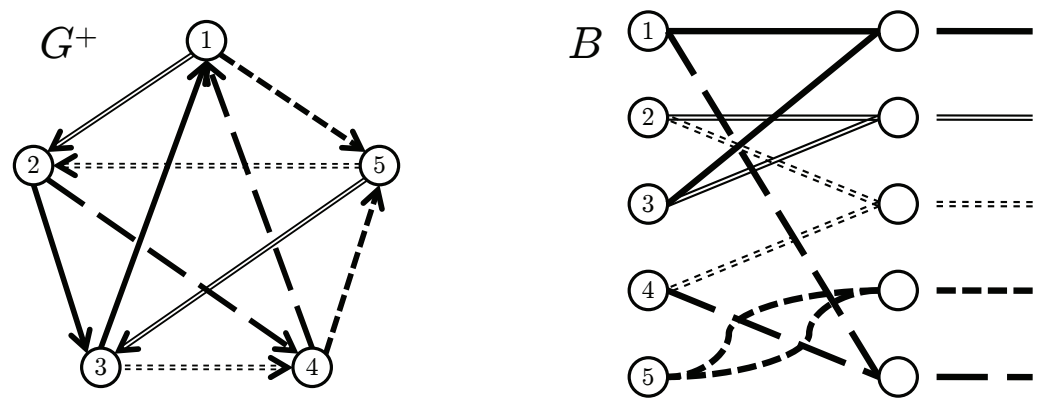

Figure 1: Example for the construction of the multigraph $B(r=2)$. The sets $E_{1}, \ldots, E_{5}$ are indicated by different types of lines.

Hence, the maximum degree in $B$ is $r$. It is well known (as Hall's theorem implies that an $r$-regular bipartite (multi)graph can be partitioned into $r$ perfect matchings) that the chromatic index of a bipartite graph equals its maximum degree. Hence there exists a proper $r$-edge-coloring $c_{B}: E(B) \rightarrow\{1, \ldots, r\}$ of $B$. Since $c_{B}$ is proper, the subsets $P_{1}^{+}, \ldots, P_{r}^{+} \subseteq E\left(G^{+}\right)$corresponding to the color classes of $c_{B}$ form a partition of $G^{+}$into $r$ edge-disjoint Achlioptas subgraphs such that the in-degree of every vertex in each of the parts is at most 1 . This implies that for $1 \leqslant i \leqslant r$ every component in $P_{i}^{+}$is a tree or unicyclic since a connected graph with two cycles has edge-density strictly larger than 1 and is thus not 1-orientable. Thus we have found a valid $r$-edge-coloring of $G^{+}$(with color classes $P_{1}^{+}, \ldots, P_{r}^{+}$) in which every monochromatic component is a tree or unicyclic, and Lemma 6 can be used to complete the proof as outlined above.

\section{References}

[1] D. Achlioptas and M. Molloy. The analysis of a list-coloring algorithm on a random graph. In Proceedings of the 38th Annual Symposium on Foundations of Computer Science, pages 204-212, 1997.

[2] T. Bohman and A. Frieze. Avoiding a giant component. Random Structures Algorithms, 19(1):75-85, 2001.

[3] T. Bohman, A. Frieze, M. Krivelevich, P.-S. Loh, and B. Sudakov. Ramsey games with giants. To appear in Random Structures Algorithms.

[4] T. Bohman, A. Frieze, and N. Wormald. Avoidance of a giant component in half the edge set of a random graph. Random Structures Algorithms, 25(4):432-449, 2004.

[5] T. Bohman and J. H. Kim. A phase transition for avoiding a giant component. Random Structures Algorithms, 28(2):195-214, 2006.

[6] T. Bohman and D. Kravitz. Creating a giant component. Combin. Probab. Comput., 15(4):489-511, 2006.

[7] B. Bollobás. Random graphs, volume 73 of Cambridge Studies in Advanced Mathematics. Cambridge University Press, Cambridge, second edition, 2001. 
[8] J. Cain, P. Sanders, and N. Wormald. The random graph threshold for $k$-orientability and a fast algorithm for optimal multiple-choice allocation. In Proceedings of the Eighteenth Annual ACM-SIAM Symposium on Discrete Algorithms, pages 469-476, New York, 2007. ACM.

[9] J. Cain and N. Wormald. Encores on cores. Electron. J. Combin., 13(1):Research Paper 81, 13 pp. (electronic), 2006.

[10] P. Erdős and A. Rényi. On the evolution of random graphs. Magyar Tud. Akad. Mat. Kutató Int. Közl., 5:17-61, 1960.

[11] D. Fernholz and V. Ramachandran. The $k$-orientability thresholds for $G_{n, p}$. In Proceedings of the Eighteenth Annual ACM-SIAM Symposium on Discrete Algorithms, pages 459-468, New York, 2007. ACM.

[12] A. Flaxman, D. Gamarnik, and G. Sorkin. Embracing the giant component. Random Structures Algorithms, 27(3):277-289, 2005.

[13] N. Fountoulakis. Thresholds and the Structure of Sparse Random Graphs. D.phil. thesis, University of Oxford, 2003.

[14] S. Janson, T. Łuczak, and A. Ruciński. Random graphs. Wiley-Interscience, New York, 2000.

[15] B. Pittel, J. Spencer, and N. Wormald. Sudden emergence of a giant $k$-core in a random graph. J. Combin. Theory Ser. B, 67(1):111-151, 1996.

[16] J. Spencer and N. Wormald. Birth control for giants. Combinatorica, 27(5):587-628, 2007.

[17] R. Sphel, A. Steger, and H. Thomas. Coloring the edges of a random graph without a monochromatic giant component. Electronic Notes in Discrete Mathematics, 34:615619, 2009. European Conference on Combinatorics, Graph Theory and Applications (EuroComb 2009). 